\title{
Confiabilidad interevaluador del K-SADS-PL-2009/trastornos del espectro autista (TEA)
}

\author{
Patricia Zavaleta-Ramírez, ${ }^{1}$ Omar Náfate-López, ${ }^{2}$ Gabriela Villarreal-Valdés, ${ }^{3}$ \\ Rosa Elena Ulloa-Flores, ${ }^{4}$ Lilia Albores-Gallo ${ }^{4}$
}

Artículo original

\section{SUMMARY}

Autism spectrum disorders (ASD) have demonstrated an increase in prevalence and are no longer considered a rare condition. The Autism Diagnostic Interview-Revised (ADI-R) and the Autism Diagnostic Observation Schedule-Generic (ADOS-G) have been gold standard instruments to diagnose ASD, but neither of these tools identifies associated comorbidity. The new Kiddie Schedule for Affective Disorders and Schizophrenia for School-Age Present and Life time version (K-SADS-PL-2009) added a supplement for assessing ASD.

\section{Objective}

The purpose of this study was to translate, adapt and analyze the inter-rater reliability of the KIDDIE SADS-PL-2009/ASD.

\section{Method}

The sample consisted of 40 children and adolescents, both male and female, with an age range between four and 17 years old, and presumptive ASD diagnosis. The original ASD screen and supplement interview was translated in to Spanish by two of the authors and retranslated by a certified translator blind to the study.

\section{Results}

Inter-rater reliability: Intraclass correlation coefficients varied from moderate to excellent for the following diagnosis both in the present and the past: Autism 0.79, 0.74; Asperger 0.85, 1.0; PDDNOS $0.72,0.41$. Kappa coefficients for expert evaluations in the present and the past were as following: Autism 0.89, 0.87; Asperger 0.77, 1.00; PDDNOS 0.69, 0.64. Diagnostic concordance between the initial clinical diagnosis and the following diagnosis through the interview was: $37.5 \%$, with a $67.5 \%$ error margin.

\section{Conclusions}

In this study, the K-SADS-PL-2009/ASD showed moderate inter-rater reliability indicating that it can be used for clinical or research purposes in Mexican children and adolescents.
Key words: Validity, reliability, K-SADS, autism, autism spectrum disorders.

\section{RESUMEN}

El incremento en la prevalencia de los trastornos del espectro autista (TEA) provocó que dejaran de considerarse como poco frecuentes. La Entrevista Diagnóstica de Autismo Revisada (ADI-R; Autism Diagnostic Interview-Revised) y la Cédula de Observación General para el Diagnóstico del Autismo (ADOS-G, Autism Diagnostic Observation Schedule-Generic) se consideran estándares de oro para diagnósticar TEA. Sin embargo, ninguno de estos instrumentos evalúa la comorbilidad asociada. La nueva versión de la Entrevista de Diagnóstico Psiquiátrico para Niños y Adolescentes, K-SADS-PL-2009, agregó un apartado para el diagnóstico de los TEA.

\section{Objetivo}

El propósito de este estudio fue traducir, adaptar y analizar la confiabilidad interevaluadora del K-SADS-PL-2009/TEA.

\section{Método}

La muestra se conformó por 40 niños y adolescentes, de ambos sexos, con un rango de edad de cuatro a 17 años, con un diagnóstico presuntivo de trastorno del espectro autista. La versión original del K-SADS-PL-2009/TEA fue traducida al español por dos de los autores y retraducida al inglés por un traductor certificado ciego al estudio.

\section{Resultados}

Confiabilidad interevaluador. Los coeficientes de correlación intraclase fueron de buenos a excelentes para los siguientes diagnósticos en el presente y pasado: autismo 0.79 y 0.74 ; trastorno de Asperger 0.85 y 1.0 ; trastorno generalizado del desarrollo no especificado (TGDNE) 0.72 y 0.41 . Los coeficientes kappa para

\section{Dirección de Servicios Clínicos, Instituto Nacional de Psiquiatría Ramón de la Fuente Muñiz.}

2 Unidad de Neuropsiquiatría Infantil Dr. Manuel Velasco Suárez. Hospital de Especialidades Pediátricas, Centro Regional de Alta Especialidad. Tuxtla Gutiérrez, Chiapas, México.

3 Centro Estatal de Salud Mental Martín Enrique y Juan O’Donojú, Saltillo, Coahuila, México.

4 Hospital Psiquiátrico Infantil Juan N. Navarro.

Correspondencia: Lilia Albores Gallo. División de Investigación, Unidad de Epidemiología Clínica, Comunitaria y Genética, Hospital Psiquiátrico Infantil Juan N. Navarro, Secretaría de Salud. Av. San Buenaventura 86, Belisario Domínguez, Tlalpan, 14080, México DF. Tel: 5573 - 4866 ext. 105,281 y 260. Cel: (044-55) 2316 - 2517. E-mail: lilialbores@gmail.com; lilialbores@yahoo.com.mx

Recibido primera versión: 16 de junio de 2014. Segunda versión: 18 de agosto de 2014. Aceptado: 1 de septiembre de 2014. 
las evaluaciones realizadas por los expertos fueron de buenos a excelentes para los siguientes diagnósticos en el presente y en el pasado: autismo 0.89 y 0.87 ; Asperger 0.77 y 1.00 ; TGDNE 0.69 y 0.64 . La concordancia entre el diagnóstico realizado en el servicio de urgencias y el corroborado posteriormente por medio del instrumento diagnóstico fue de $37.5 \%$, con un índice de error diagnóstico de $67.5 \%$.

\section{Conclusiones}

El K-SADS-PL-2009/TEA mostró una buena confiabilidad interevaluador y puede usarse en niños y adolescentes mexicanos para propósitos clínicos y de investigación.

Palabras clave: Validez, confiabilidad, KIDDIE SADS, autismo, trastornos del espectro autista.

\section{INTRODUCCIÓN}

El diagnóstico correcto y temprano del autismo es importante pues se sabe que la intervención oportuna con técnicas de análisis conductual aplicado mejora el pronóstico de los niños. ${ }^{1}$ Aunque en las últimas dos décadas se ha promovido el desarrollo de instrumentos para evaluar los trastornos del espectro autista orientados al tamizaje, diagnóstico y detección de cambios (Albores et al., 2008), ${ }^{2}$ pocas de estas herramientas se encuentran disponibles para población mexicana. La validación del M-CHAT realizada por Albores et al. en $2012^{3}$ y del ABC por Varela y Albores en $2012^{4}$ muestran que la equivalencia transcultural de los instrumentos es difícil de alcanzar por motivos como sesgos culturales, como reconocen algunos autores. ${ }^{5-7}$ Por eso no basta con traducir los instrumentos: éstos deben adaptarse culturalmente para utilizarse de manera eficiente.

Aunque existen entrevistas para realizar un diagnóstico de certeza de los trastornos del espectro autista, como la Cédula de Observación General para el Diagnóstico del Autismo (ADOS-G, Autism Diagnostic Observation Schedule-Generic) $)^{8}$ y la Entrevista Diagnóstica de Autismo Revisada (ADI-R, Autism Diagnostic Interview revised), ${ }^{9}$ estas herramientas tienen el inconveniente de que su aplicación es larga, requieren entrenamiento y son de uso reservado a personal con mucha experiencia en este campo. Además, no evalúan la comorbilidad psiquiátrica, que es muy elevada en los TEA. De igual manera, entrevistas psiquiátricas, como la Entrevista de evaluación psiquiátrica para el niño y el adolescente (CAPA, Child and Adolescent Psychiatric Assessment), de Angold y Costello, ${ }^{10}$ y la más reciente versión de la Entrevista para la evaluación psiquiátrica del niño en edad preescolar (PAPA, Preschool Age Psychiatric Assessment), Egger et al., 2006, ${ }^{11}$ no incluyen reactivos para evaluar los problemas del desarrollo. La consecuencia es que el autismo no se estudia en las encuestas de psiquiatría y que hay pocos estudios sobre comorbilidad psiquiátrica y seguimiento longitudinal en menores con autismo. ${ }^{12}$

A pesar de no contener una sección específica para evaluar los síntomas de autismo, el K-SADS-PL fue usado por Ivarzon, en 2001,13 y Sinzing, en 2008,,$^{14}$ para estudiar la asociación de trastornos psiquiátricos en niños con espectro autista (TEA). Por su parte, Lee la utilizó en $2006^{15}$ para evaluar a pacientes con trastorno por déficit de atención con hi- peractividad y su relación con los trastornos generalizados del desarrollo no especificados (TGDNE).

En 2006 Leyfer realizó una modificación a toda la entrevista K-SADS-PL, nombrándola Entrevista de Comorbilidad del Autismo, versión actual, y a lo largo de la vida (ACI-PL, Autism Comorbidity Interview-Present and Lifetime Version) ${ }_{1}^{16}$ para estudiar la discapacidad asociada al autismo. Estos estudios evidenciaron la necesidad de que el K-SADS-PL, instrumento que evalúa la psicopatología y el funcionamiento a lo largo de la vida, contuviera un apartado para evaluar a niños y adolescentes con trastornos del espectro autista.

En México, Ulloa et al. validaron en $2006^{17}$ el K-SADSPL, mostrando excelente confiabilidad interevaluador con índices kappa mayores para el trastorno depresivo mayor, 0.76; trastorno distímico, 0.77 ; trastorno por déficit de atención con hiperactividad, 0.91 ; y trastorno oposicionista desafiante, 0.71. Sin embargo, no fue sino hasta 2009 en que se agregó al K-SADS-PL un apartado para evaluar los trastornos del espectro autista. A diferencia del ADI-R y el ADOS-G, este apartado contiene algoritmos para el diagnóstico de autismo, trastorno de Asperger y TGDNE, y no requiere entrenamiento. Asimismo, su tiempo de administración más corto permite la observación del menor con TEA al tiempo que se entrevista al padre o tutor.

El objetivo de este estudio fue evaluar la confiabilidad interevaluador de la entrevista diagnóstica del K-SADS-PL2009/TEA (tamizaje y suplemento) en niños y adolescentes mexicanos.

\section{MATERIAL Y MÉTODO}

\section{Participantes}

Se incluyó a 40 niños y adolescentes de ambos sexos, de entre cuatro y 17 años de edad, reclutados en la consulta externa de un hospital de psiquiatría infantil, con diagnóstico presuntivo de trastorno del espectro autista. Después de conocer el propósito del estudio los padres firmaron el consentimiento informado y los adolescentes dieron su asentimiento. Se excluyó a los participantes con algún trastorno psicótico. Se eliminó a los participantes que no completaron de forma correcta los cuestionarios. 


\section{Instrumentos de medición}

Entrevista semiestructurada para evaluar los trastornos del espectro autista con base en los criterios del DSM-IV en los tiempos presente y pasado. Esta entrevista se ha usado antes como estándar de oro y ha mostrado su efectividad.

Schedule for Affective Disorders and Schizophrenia for School-Age Children (K-SADS-PL). ${ }^{18}$ Es una entrevista semiestructurada que evalúa psicopatología de manera categórica de acuerdo con los criterios diagnósticos del DSMIV. ${ }^{19}$ Permite establecer la edad de inicio y/o la remisión de los síntomas en el presente y a lo largo de la vida. Se diseñó para evaluar a niños y adolescentes de entre seis y 17 años, aunque Birmaher demostró en 2009 su uso y validez en menores de seis años. ${ }^{20}$

El instrumento consta de tres secciones: 1. Entrevista introductoria que recoge datos de la historia médica, escolar, familiar, así como del funcionamiento global (C-GAS). 2. La sección de tamizaje consta de dos o tres preguntas que evalúan síntomas clave. Las respuestas se codifican de la siguiente manera: $0=\sin$ información, $1=$ ausente, $2=$ presente y $3=$ umbral. 3. La sección del suplemento diagnóstico se administra cuando cualquier síntoma de la sección de tamizaje se codifica como umbral=3 en el sumario del clínico.

Las respuestas de los informantes (padre y niño/adolescente) se registran de manera independiente. El entrevistador elabora un sumario que es el mejor estimado clínico a partir del informe del padre, del menor y de la observación directa del paciente.

La sección de tamizaje cuenta con cinco preguntas que evalúan los manierismos motores repetitivos y estereotipados, la adherencia inflexible a rutinas o rituales, la preocupación persistente por intereses restringidos/estereotipados, el deterioro en el comportamiento no verbal, como la mirada, las expresiones y/o los gestos y el retraso en el lenguaje. Este último se codifica como "umbral" cuando las palabras sueltas o el uso de frases se expresan tres meses más tarde de lo esperado ( $<24$ meses de edad y las frases a los 33 meses). Además de este reactivo, se redactó otro para evaluar el retraso en el desarrollo del lenguaje cuando el niño no expresa 50 palabras sueltas a los 24 meses, o usa frases a los 27 meses o después. ${ }^{21} \mathrm{Si}$ el niño carece de lenguaje, sus respuestas se codifican como "sin información".

Asimismo, el suplemento evalúa la falta de motivación para compartir disfrutes, intereses o logros con los demás y la incapacidad para desarrollar relaciones apropiadas con compañeros de la misma edad. Además se explora la reciprocidad social o emocional y la preocupación persistente por partes de objetos. La última sección examina las deficiencias sociales y de lenguaje, como el déficit pragmático, la prosodia, la ecolalia, la verbosidad, el aislamiento social y las habilidades de autoayuda en pacientes con trastorno de Asperger. También evalúa el deterioro funcional.
El diagnóstico final se considera definitivo si se cumplen los criterios del DSM-IV probable cuando se cumple el $75 \%$ de los criterios diagnósticos de un trastorno o ausente cuando no se cumplen.

Entrevista Diagnóstica de Autismo Revisada (ADI-R, Autism Diagnostic Interview-Revised) (Lord et al., 1994). ${ }^{9}$ Este instrumento se considera como criterio de oro para diagnosticar autismo. Es una entrevista semiestructurada administrada por clínicos con amplia experiencia en autismo, para evaluar síntomas del trastorno a lo largo del desarrollo. Los resultados se organizan en tres dominios: $a$ ) déficit de reciprocidad social, $b$ ) problemas de comunicación y c) conducta e intereses restrictivos y estereotipados. El ADI-R se basa en la frecuencia de síntomas a lo largo de la vida con énfasis especial en los cuatro o cinco años de edad. Este instrumento discrimina a los niños con autismo de otros con trastornos psiquiátricos y con desarrollo típico. ${ }^{22-24}$ Es más preciso con niños mayores a 40 meses de edad como lo demostró Cox en 1999,22 y posee mayor validez para identificar autismo que síndrome de Asperger ${ }^{23}$ (Gilchrist et al., 2001). ${ }^{25}$

Cicchetti et al. (2008) ${ }^{26}$ estudiaron la confiabilidad del ADI-R y encontraron coeficientes de correlación elevados (94-96\%) en todos los reactivos, con kappas (K) entre .80 y .88 y .40 y .47 .

\section{PROCEDIMIENTO}

La versión original del tamizaje y suplemento del K-SADSPL-2009/TEA fue traducida al español por dos de los autores y posteriormente la retradujo al inglés un traductor certificado ciego al estudio. La validez de apariencia y de contenido de la versión en español se evaluó mediante la opinión de tres expertos en salud mental. Antes de usar formalmente el instrumento se realizaron 12 entrevistas piloto para valorar aspectos de la entrevista como la fluidez y la comprensión por parte de los padres y de los niños y adolescentes.

El reclutamiento de los participantes se llevó a cabo en el Hospital Psiquiátrico Infantil Juan N. Navarro de mayo de 2010 a enero de 2011. Los médicos de la consulta externa del hospital refirieron a aquellos niños con diagnóstico presuntivo de autismo, trastorno de Asperger y TGDNE. Después de explicárseles el objetivo del estudio, los padres firmaron la carta de consentimiento informado y los adolescentes y niños mayores de ocho años dieron su asentimiento para participar. La aplicación de las entrevistas K-SADSPL/TEA (tamizaje y suplemento) estuvo a cargo de dos expertos: una psicóloga y un paidopsiquiatra, y dos residentes en psiquiatría infantil y de la adolescencia que recibieron entrenamiento formal durante tres meses. Un grupo de residentes independiente al estudio aplicó la entrevista semiestructurada que evalúa las subcategorías de trastorno autista, trastorno de Asperger y TGDNE en tiempo presente y pasado. 


\section{Implicaciones éticas}

El presente estudio fue aprobado por el Comité de Ética del hospital participante. El consentimiento informado se obtuvo de cada uno de los padres de los niños y/o adolescentes participantes.

\section{Análisis estadístico}

Análisis de variables demográficas. Se utilizaron medidas de tendencia central para describir las variables demográficas. Así se reportaron frecuencias para las variables categóricas, medias y desviaciones estándar para las variables continuas. Para evaluar la confiabilidad de la entrevista (tamizaje y suplemento), se utilizó el coeficiente de correlación intraclase. Además, se calculó el coeficiente kappa de Cohen para evaluar la confiabilidad entre los dos expertos. Se consideró una excelente confiabilidad cuando el valor del coeficiente kappa fue mayor que 0.75 ; buena si oscilaba entre $0.59 \mathrm{y}$ 0.74 ; moderada de 0.40 a 0.58 , y pobre si era menor que 0.40 . Finalmente, se calculó el porcentaje de acuerdo con el diagnóstico realizado en el servicio de urgencias y el diagnóstico obtenido por medio de la entrevista.

\section{RESULTADOS}

Se reclutó a un total de 45 participantes, de los cuales se excluyó a aquéllos que no llenaron el formato de datos sociodemográficos y/o aquellos de quienes un evaluador consideró poco confiable la información proporcionada durante la entrevista diagnóstica de los criterios del DSM-IV y/o el ADI-R. El grupo que se consideró para el análisis $(\mathrm{N}=40)$ tuvo una edad promedio 8.96 años (intervalo de 3.0 a 17.1 años); $80 \%(n=32)$ fueron del sexo masculino.

\section{Confiabilidad interevaluador}

Los coeficientes de correlación intraclase fueron de buenos a excelentes para la mayoría de los diagnósticos (cuadro 1). Los coeficientes kappa entre las evaluaciones

Cuadro 1. Confiabilidad interevaluador para los diferentes diagnósticos de trastornos del espectro autista de la entrevista diagnóstica K-SADS-PL 2009

\begin{tabular}{lcc}
\hline Diagnóstico K-SADS-PL & $\begin{array}{c}\text { Correlación intraclase } \\
(\mathrm{n}=40)\end{array}$ & $\mathrm{IC}$ \\
\hline Autismo presente & .79 & $.70-.87$ \\
Autismo pasado & .74 & $.62-.84$ \\
Asperger presente & .85 & $.74-.93$ \\
Asperger pasado & 1.00 & $1.0-1.0$ \\
TGDNE presente & .72 & $.60-.82$ \\
TGDE pasado & .41 & $.25-.58$ \\
\hline
\end{tabular}

IC = Intervalo de confianza.
Cuadro 2. Coeficientes kappa entre expertos de los diagnósticos obtenidos de la entrevista diagnóstica K-SADS-PL 2009 TEA

\begin{tabular}{lc}
\hline Diagnóstico K-SADS-PL & Correlación Kappa \\
\hline Autismo presente & .89 \\
Autismo pasado & .87 \\
Asperger presente & .77 \\
Asperger pasado & 1.00 \\
TGDNE presente & .69 \\
TGDNE pasado & .64 \\
\hline
\end{tabular}

realizadas por los expertos también fueron de buenos a excelentes (cuadro 2). Finalmente, la coincidencia entre el diagnóstico realizado en el servicio de urgencias y el corroborado por medio del instrumento diagnóstico fue de $37.5 \%$, con un índice de error diagnóstico de $67.5 \%$.

\section{DISCUSIÓN}

En este estudio se evaluó la confiabilidad interevaluador de la entrevista diagnóstica K-SADS-PL-2009/TEA para trastornos del espectro autista. A diferencia del ADI-R y el ADOS-G, el apartado TEA del K-SADS permite el diagnóstico diferenciado del autismo, del trastorno de Asperger y del TGDNE, según los criterios del DSM-IV. En la reciente edición del DSM- $5,{ }^{27}$ estas categorías del DSM-IV se integran en una sola categoría de TEA, con el propósito de aumentar la confiabilidad y el valor predictivo del pronóstico, que la categoría determinada en la clasificación del DSM-IV..$^{28,29}$

Nuestros resultados mostraron que el apartado de TEA tuvo una confiabilidad interevaluador buena para las subcategorías. Los coeficientes kappa para autismo fueron superiores entre los evaluadores expertos, donde destaca la importancia de la experiencia clínica para evaluar los TEA como señalan algunos investigadores. ${ }^{30}$

La elevada concordancia para el diagnóstico de Asperger en "el pasado" correspondió con el uso del criterio cuantitativo para identificar el retraso en el desarrollo de lenguaje (no expresa palabras $<24$ meses de edad y frases a los 33 meses, y/o expresa $\leq 50$ palabras a los 24 meses o usa frases a los 27 meses), cuyo valor umbral de tres en el tamizaje distingue al autismo del trastorno de Asperger. El número de palabras que el niño expresa a los 24 meses es una variable muy importante para el diagnóstico de los TEA, demostró Jeans en 2014 quien estableció que un punto de corte de $\leq 25$ palabras a los dos años de edad diferenció correctamente el autismo del trastorno de Asperger. ${ }^{31}$ Este criterio es inferior al propuesto por nosotros de $\leq 50$ palabras a los dos años de edad y debería tomarse en cuenta en estudios futuros.

A pesar de la importancia de la presencia/ausencia del retraso del desarrollo del lenguaje como criterio útil para el 
diagnóstico de los TEA y de las subcategorías, éste se eliminó del DSM-5, pues se dio más énfasis a los problemas semántico-pragmáticos del lenguaje. Es probable que este hecho disminuya la identificación temprana del autismo, ya que el retraso de lenguaje es la causa principal de búsqueda de atención médica por parte de los padres, como muestran diversos estudios. 32,33

Aunque todas las preguntas de la entrevista deben formularse al niño y/o adolescente, en nuestro estudio, $90 \%$ de ellos fueron informantes poco confiables. Este resultado es consistente con el estudio de Mazefsky (2011), quien demostró una concordancia baja entre las respuestas de los adolescentes con TEA por encima de los síntomas de depresión y ansiedad y las respuestas del padre por medio de la entrevista ACI-PL. ${ }^{34}$

A pesar de esto, el apartado TEA de la entrevista K-SADS-PL demostró que la información proporcionada por el padre, la observación directa del niño o adolescente y la experiencia del clínico fueron elementos suficientes para establecer un mejor estimado, cuyo diagnóstico tuvo una confiabilidad interevaluador de moderada a buena.

Estudios futuros deben investigar la validez de constructo de esta entrevista para diagnosticar los trastornos del espectro autista en sujetos de distintas edades y con diferentes niveles de funcionamiento.

El K-SADS-PL/TEA entraña algunas limitaciones, entre las que podemos mencionar el hecho de que no contiene una sección para evaluar la pérdida de habilidades de lenguaje o motoras como el ADI-R y que permite clasificar el autismo en su variedad regresiva. Tampoco evalúa las habilidades especiales de los individuos con TEA que son características de los individuos denominados savants. Debido a que la entrevista se diseñó antes de la publicación del DSM-5, su compatibilidad con esta clasificación es parcial ya que no explora la hipersensibilidad sensorial, que es un criterio añadido al DSM-5.

Este estudio tiene limitaciones importantes entre las que podemos mencionar el reducido tamaño de la muestra y el no contar con una medida de coeficiente intelectual para probar el efecto de éste sobre los reactivos que miden el retraso en el lenguaje, que es muy común en la discapacidad intelectual..$^{35}$ A pesar de estas limitaciones, el apartado TEA del K-SADS-PL es un instrumento útil que integra el informe del desarrollo del menor por medio de la entrevista con el padre y la observación del niño o adolescente. Esta información recabada en un solo instrumento es una ventaja si se compara con el costo y tiempo de administrar los instrumentos ADOS-G (observacional) y el ADI-R (entrevista del padre), cuya combinación es la que arroja los mejores resultados de sensibilidad y especificidad como sugieren diversos autores. ${ }^{36,37}$

Además, el K-SADS-PL/TEA es de administración sencilla y breve, por lo que es ideal para usarse en ambientes clínicos saturados.

\section{AGRADECIMIENTOS}

Los autores agradecen la cooperación de todas las familias participantes en este estudio.

\section{REFERENCIAS}

1. Dawson G. Early behavioral intervention, brain plasticity, and the prevention of autism spectrum disorder. Dev Psychopathol 2008;20:775803.

2. Albores-Gallo L, Hernández-Guzmán L, Díaz-Pichardo JA, Cortes-Hernández B. Dificultades en la evaluación y diagnóstico del autismo: Una discusión. Salud Mental 2008;31:37-44.

3. Albores-Gallo L, Roldán-Ceballos O, Villarreal-Valdés G, Betanzos-Cruz BX et al. M-CHAT Mexican version validity and reliability and some cultural considerations. ISRN Neurol 2012:408694.

4. Living-Varela K. Confiabilidad y validez del inventario de comportamiento autista $(\mathrm{ABC})$ en niños con trastornos del espectro autista. 2012. Available at: https://sites.google.com/site/hpicomisioninvestigacion/psiquiatria-infantil-y-de-la-adolescencia.

5. Robins DL. Screening for autism spectrum disorders in primary care settings. Autism Int J Res Pract 2008;12:537-556.

6. Inada N, Koyama T, Inokuchi E, Kuroda M et al. Reliability and validity of the Japanese version of the Modified Checklist for autism in toddlers (M-CHAT). Res Autism Spectr Disord 2011;5:330-336.

7. Wong V, Hui L-HS, Lee W-C, Leung L-SJ et al. A modified screening tool for autism (Checklist for Autism in Toddlers [CHAT-23]) for Chinese children. Pediatrics 2004;114:e166-176.

8. Lord C, Risi S, Lambrecht L, Cook Jr EH et al. The Autism Diagnostic Observation Schedule-Generic: A standard measure of social and communication deficits associated with the spectrum of autism. J Autism Dev Disord 2000;30:205-223.

9. Lord C, Rutter M, Couteur A Le. Autism Diagnostic Interview-Revised: a revised version of a diagnostic interview for caregivers of individuals with possible pervasive developmental disorders. J Autism Dev Disord 1994;24:659-685.

10. Angold A, Costello EJ. The Child and Adolescent Psychiatric Assessment (CAPA). J Am Acad Child Adolesc Psychiatry 2000;39:39-48.

11. Egger HL, Erkanli A, Keeler G, Potts E et al. Test-Retest Reliability of the Preschool Age Psychiatric Assessment (PAPA). J Am Acad Child Adolesc Psychiatry 2006;45:538-549.

12. Gjevik E, Eldevik S, Fjæran-Granum T, Sponheim E. Kiddie-SADS reveals high rates of DSM-IV disorders in children and adolescents with autism spectrum disorders. J Autism Dev Disord 2010;41:761-769.

13. Ivarsson T, Melin K. Autism spectrum traits in children and adolescents with obsessive-compulsive disorder (OCD). J Anxiety Disord 2008;22:969-978.

14. Sinzig J, Walter D, Doepfner M. Attention Deficit/hyperactivity disorder in children and adolescents with autism spectrum disorder: Symptom or syndrome? J Atten Disord 2009;13:117-126.

15. Lee DO, Ousley OY. Attention-deficit hyperactivity disorder symptoms in a clinic sample of children and adolescents with pervasive developmental disorders. J Child Adolesc Psychopharmacol 2006;16:737746.

16. Leyfer OT, Folstein SE, Bacalman S, Davis NO et al. Comorbid psychiatric disorders in children with autism: interview development and rates of disorders. J Autism Dev Disord 2006;36:849-861.

17. Ulloa RE, Ortiz S, Higuera F, Nogales I et al. [Interrater reliability of the Spanish version of schedule for affective disorders and schizophrenia for school-age children-present and lifetime version (K-SADS-PL)]. Actas Esp Psiquiatr 2006;34:36-40.

18. Kaufman J, Birmaher B, Brent D, Rao U et al. Schedule for affective disorders and schizophrenia for school-age children-present and lifetime version (K-SADS-PL): initial reliability and validity data. J Am 
Acad Child Adolesc Psychiatry 1997;36:980-988.

19. American Psychiatric Association. Diagnostic and statistical manual of mental disorders. Washington, DC.: 2000.

20. Birmaher B, Ehmann M, Axelson DA, Goldstein BI et al. Schedule for affective disorders and schizophrenia for school-age children (K-SADS-PL) for the assessment of preschool children-a preliminary psychometric study. J Psychiatr Res 2009;43:680-686.

21. Rescorla L, Alley A. Validation of the language development survey (LDS): a parent report tool for identifying language delay in toddlers. J Speech Lang Hear Res JSLHR 2001;44:434-445.

22. Cox A, Klein K, Charman T, Baird G et al. Autism spectrum disorders at 20 and 42 months of age: stability of clinical and ADI-R diagnosis. J Child Psychol Psychiatry 1999;40:719-732.

23. Gilchrist A, Green J, Cox A, Burton D et al. Development and current functioning in adolescents with Asperger syndrome: a comparative study. J Child Psychol Psychiatry 2001;42:227-240.

24. Lord C, Rutter M, Couteur A Le. Autism Diagnostic Interview-Revised: a revised version of a diagnostic interview for caregivers of individuals with possible pervasive developmental disorders. J Autism Dev Disord 1994;24:659-685.

25. Gilchrist A, Green J, Cox A, Burton D et al. Development and current functioning in adolescents with Asperger syndrome: a comparative study. J Child Psychol Psychiatry 2001;42:227-240.

26. Cicchetti DV, Lord C, Koenig K, Klin A et al. Reliability of the ADI-R: multiple examiners evaluate a single case. J Autism Dev Disord 2008;38:764-770.

27. American Psychiatric Association AP. Diagnostic and Statistical Manual of Mental Disorders. Quinta edición. Washington, D.C: American Psychiatric Publishing; 2013.

28. Szatmari P, Bryson SE, Boyle MH, Streiner DL et al. Predictors of outcome among high functioning children with autism and Asperger syndrome. J Child Psychol Psychiatry 2003;44:520-528.
29. Szatmari P, Bryson S, Duku E, Vaccarella L et al. Similar developmental trajectories in autism and Asperger syndrome: from early child-hood to adolescence. J Child Psychol Psychiatry 2009;50:1459-1467.

30. Reaven JA, Hepburn SL, Ross RG. Use of the ADOS and ADI-R in children with psychosis: importance of clinical judgment. Clin Child Psychol Psychiatry 2008;13:81-94.

31. Jeans LM, Santos RM, Laxman DJ, McBride BA et al. Early predictors of ASD in young children using a nationally representative data set. J Early Interv 2014. Available at: http://jei.sagepub.com/cgi/ doi/10.1177/1053815114523319. Última visita el 12 de agosto, 2014.

32. Herlihy L, Knoch K, Vibert B, Fein D. Parents' first concerns about toddlers with autism spectrum disorder: Effect of sibling status. Autism Int J Res Pract 2013.

33. Kozlowski AM, Matson JL, Horovitz M, Worley JA et al. Parents' first concerns of their child's development in toddlers with autism spectrum disorders. Dev Neurorehabilitation 2011;14:72-78.

34. Mazefsky CA, Kao J, Oswald DP. Preliminary evidence suggesting caution in the use of psychiatric self-report measures with adolescents with high-functioning autism spectrum disorders. Res Autism Spectr Disord 2011;5:164-174.

35. Emerson E, Brigham P. The developmental health of children of parents with intellectual disabilities: cross sectional study. Res Dev Disabil 2014;35:917-921.

36. Bildt A de, Sytema S, Ketelaars C, Kraijer D et al. Interrelationship between Autism Diagnostic Observation Schedule-Generic (ADOS-G), Autism Diagnostic Interview-Revised (ADI-R), and the Diagnostic and Statistical Manual of Mental Disorders (DSM-IV-TR) classification in children and adolescents with mental retardation. J Autism Dev Disord 2004;34:129-137.

37. Bölte S, Poustka F. [Diagnostic Observation Scale for Autistic Disorders: initial results of reliability and validity]. Z Für Kinder- Jugendpsychiatrie Psychother 2004;32:45-50.

Artículo sin conflicto de intereses 